\title{
Measure of departure from average marginal homogeneity for the analysis of collapsed ordinal square contingency tables
}

\author{
Mana Aizawa ${ }^{1}$, Kouji Yamamoto ${ }^{2}$ and Sadao Tomizawa ${ }^{3}$ \\ ${ }^{1}$ Department of Information Sciences, Faculty of Science and Technology, Tokyo \\ University of Science, Noda City, Chiba, 278-8510, Japan, \\ e-mail: aizawa.m01@gmail.com \\ ${ }^{2}$ Department of Biostatistics, School of Medicine, Yokohama City University, 3-9 \\ Fukuura, Kanazawa-ku, Yokohama, 236-0004, Japan \\ e-mail: kouji_y@yokohama-cu.ac.jp \\ ${ }^{3}$ Department of Information Sciences, Faculty of Science and Technology, Tokyo \\ University of Science, Noda City, Chiba, 278-8510, Japan, \\ e-mail: tomizawa@is.noda.tus.ac.jp
}

\begin{abstract}
SUMmarY
In clinical research, collected data are often classified into ordered categories using a set threshold to evaluate efficacy and safety of treatment. Data can be summarized as a shift table, which displays the change in the frequency of subjects across specified categories from the baseline to post-baseline. Although ordered categories are sometimes recombined into three categories, the combined patterns vary. To consider various collapsed patterns comprehensively, this paper proposes a new measure that represents the degree of departure from average marginal homogeneity, and can distinguish between two kinds of marginal inhomogeneity. Additionally, applications of the proposed measure to clinical data are discussed.
\end{abstract}

Key words: Collapsed table, marginal homogeneity, measure

\section{Introduction}

In clinical research, collected data are often classified into ordered categories using a set threshold to evaluate efficacy and safety of treatment. In particular, laboratory data are often classified into categorical values using predetermined thresholds. Table 1, which is taken from Nogid et al. (2018), shows an example. These data represent shifts in renal function categories 
from the baseline to the end of therapy in patients treated with telavancin or vancomycin. The row values and column values describe the creatinine clearance $(\mathrm{CrCl})$ level at the end of treatment and at the baseline, respectively. For such data, it is interesting to evaluate the equality of the row and column marginal distributions.

Table 1. Shifts in the renal function categories from the baseline to the end of therapy in patients treated with (a) telavancin and (b) vancomycin; taken from Nogid et al. (2018)

\begin{tabular}{lccccccccc}
\hline \multirow{2}{*}{$\begin{array}{c}\text { End of therapy CrCl } \\
\text { category (mL/min) }\end{array}$} & \multicolumn{7}{c}{ Baseline CrCl category $(\mathrm{mL} / \mathrm{min})$} \\
\cline { 3 - 10 } \multicolumn{1}{c}{} & $(1)$ & $(2)$ & $(3)$ & $(4)$ & $(5)$ & $(6)$ & $(7)$ & Total \\
\hline (a) Telavancin & $(1)$ & 60 & 15 & 8 & 11 & 5 & 6 & 4 & 109 \\
& $(2)$ & 8 & 21 & 10 & 6 & 3 & 1 & 9 & 58 \\
& $(3)$ & 6 & 12 & 19 & 11 & 5 & 4 & 3 & 60 \\
& $(4)$ & 1 & 5 & 16 & 15 & 7 & 5 & 12 & 61 \\
& $(5)$ & 2 & 4 & 8 & 10 & 13 & 8 & 12 & 57 \\
& $(6)$ & 3 & 1 & 3 & 3 & 12 & 10 & 12 & 44 \\
& $(7)$ & 0 & 1 & 7 & 3 & 16 & 12 & 256 & 295 \\
\hline & Total & 80 & 59 & 71 & 59 & 61 & 46 & 308 & 684 \\
\hline (b) Vancomycin & $(1)$ & 50 & 10 & 2 & 1 & 4 & 2 & 7 & 76 \\
& $(2)$ & 15 & 16 & 11 & 2 & 2 & 1 & 9 & 56 \\
& $(3)$ & 9 & 24 & 11 & 10 & 4 & 1 & 10 & 69 \\
& $(4)$ & 4 & 11 & 19 & 14 & 9 & 3 & 11 & 71 \\
& $(5)$ & 3 & 5 & 8 & 6 & 12 & 6 & 11 & 51 \\
& $(6)$ & 2 & 3 & 1 & 9 & 14 & 6 & 19 & 54 \\
& $(7)$ & 0 & 3 & 9 & 13 & 15 & 32 & 249 & 321 \\
\hline & Total & 83 & 72 & 61 & 55 & 60 & 51 & 316 & 698 \\
\hline
\end{tabular}

$\mathrm{CrCl}$ indicates creatinine clearance.

$(1): \leqslant 30,(2):>30-40,(3):>40-50,(4):>50-60,(5):>60-70,(6):>70-80,(7):>80$.

To analyze the marginal homogeneity in a square contingency table which has the same row and column classifications, Stuart (1955) proposed the marginal homogeneity $(\mathrm{MH})$ model. If the $\mathrm{MH}$ model holds in Table 1, then the distribution of the $\mathrm{CrCl}$ value at the end of treatment is the same as that at the baseline.

If the MH model does not hold, one of our interests is to apply the extended models. On the other hand, measuring the degree of departure from marginal homogeneity is also of great interest. In addition, for several data sets, it is meaningful to compare the degree of departure from marginal homogeneity. For nominal square contingency tables, Tomizawa and Makii 
(2001) proposed a measure to express the degree of departure from marginal homogeneity. For ordinal square contingency tables, Tomizawa et al. (2003) proposed a measure which represents the degree of departure from marginal homogeneity. This measure ranges from 0 to 1 . The measure is 0 if and only if the MH model holds, and 1 if and only if the degree of departure from the $\mathrm{MH}$ model is maximum.

There are two kinds of departures, or marginal inhomogeneities (i.e., whether all observations concentrate in the lower left triangle cells or the upper right triangle cells in the table). These marginal inhomogeneities indicate different and opposite maximum departures from marginal homogeneity. Therefore, it is also important to distinguish between the two kinds of inhomogeneity. Yamamoto et al. (2011) proposed a measure to represent the degree of departure from average marginal homogeneity, which can distinguish between the two kinds of inhomogeneity. Ando (2019) developed an index for visually measuring the marginal inhomogeneity, which can concurrently define degrees of departure from marginal homogeneity corresponding to the extended marginal homogeneity (EMH) model (Tomizawa, 1984) and the marginal logit model (McCullagh, 1977).

To simplify the interpretation of the results, the categories for the ordinal square contingency table are often divided into three categories (e.g., mild, moderate and severe). However, for laboratory data similar to those in Table 1, various thresholds should be considered. Further, in clinical research, the data are often classified into four or more categories in guidelines. In such a case, consideration should be given to merging the categories without breaking categories which follow the original guidelines. Thus, it is also important to consider dividing the items that have already been classified into four or more categories into three categories. For the reasons above, it is meaningful to assess various collapsed patterns comprehensively. Yamamoto et al. (2020) proposed a measure for the degree of departure from marginal homogeneity for a collapsed table. However, its measure cannot distinguish between the two kinds of marginal inhomogeneity.

This paper proposes a new measure to express the degree of departure from average marginal homogeneity by adopting collapsed $3 \times 3$ tables. The proposed measure can distinguish between the two kinds of inhomogeneity. 


\section{Measure of departure from marginal homogeneity for collapsed $3 \times 3$ table}

Consider an $r \times r$ square contingency table with the same ordered row and column classifications. Let $p_{i j}$ denote the probability that an observation will fall in cell $(i, j)(i=1, \ldots, r ; j=1, \ldots, r)$. The MH model is defined as

$$
p_{i .}=p_{\cdot i} \quad \text { for } i=1, \ldots, r,
$$

where $p_{i}=\sum_{k=1}^{r} p_{i k}$ and $p_{\cdot i}=\sum_{k=1}^{r} p_{k i}$. This model indicates that the row and column marginal distributions are equal.

An $r \times r$ ordinal square contingency table can be collapsed into a $3 \times 3$ table $(r-1)(r-2) / 2$ ways by choosing cut points after the $s$ th and $t$ th rows and after the $s$ th and $t$ th columns for $1 \leqslant s<t \leqslant r-1$. Each collapsed $3 \times 3$ table is defined as $T_{s t}(1 \leqslant s<t \leqslant r-1)$. This means that $r$ categories in the original $r \times r$ table are included in three categories by dividing at the cut points $s$ and $t$. Then, the table $T_{s t}(1 \leqslant s<t \leqslant r-1)$ has the three new categories. In a collapsed table $T_{s t}(1 \leqslant s<t \leqslant r-1)$, let $G_{i j}^{(s, t)}$ indicate the corresponding probability for row value $i(i=1,2,3)$ and column value $j(j=1,2,3)$. That is,

$$
\begin{aligned}
& G_{11}^{(s, t)}=\sum_{i=1}^{s} \sum_{j=1}^{s} p_{i j}, \quad G_{12}^{(s, t)}=\sum_{i=1}^{s} \sum_{j=s+1}^{t} p_{i j}, \quad G_{13}^{(s, t)}=\sum_{i=1}^{s} \sum_{j=t+1}^{r} p_{i j}, \\
& G_{21}^{(s, t)}=\sum_{i=s+1}^{t} \sum_{j=1}^{s} p_{i j}, \quad G_{22}^{(s, t)}=\sum_{i=s+1}^{t} \sum_{j=s+1}^{t} p_{i j}, \quad G_{23}^{(s, t)}=\sum_{i=s+1}^{t} \sum_{j=t+1}^{r} p_{i j}, \\
& G_{31}^{(s, t)}=\sum_{i=t+1}^{r} \sum_{j=1}^{s} p_{i j}, \quad G_{32}^{(s, t)}=\sum_{i=t+1}^{r} \sum_{j=s+1}^{t} p_{i j}, \quad G_{33}^{(s, t)}=\sum_{i=t+1}^{r} \sum_{j=t+1}^{r} p_{i j} .
\end{aligned}
$$

The MH model can also be expressed as

$$
G_{i .}^{(s, t)}=G_{\cdot i}^{(s, t)} \quad \text { for } \quad i=1,2,3 ; 1 \leqslant s<t \leqslant r-1,
$$

where

$$
G_{i}^{(s, t)}=\sum_{j=1}^{3} G_{i j}^{(s, t)}, \quad G_{\cdot i}^{(s, t)}=\sum_{j=1}^{3} G_{j i}^{(s, t)} .
$$

Namely, the MH model can be restated as

$$
H_{1(k)}^{(s, t)}=H_{2(k)}^{(s, t)} \quad \text { for } \quad k=1,2 ; 1 \leqslant s<t \leqslant r-1,
$$


where

$$
H_{1(k)}^{(s, t)}=\sum_{u=1}^{k} \sum_{v=k+1}^{3} G_{u v}^{(s, t)}, \quad H_{2(k)}^{(s, t)}=\sum_{u=1}^{k} \sum_{v=k+1}^{3} G_{v u}^{(s, t)} .
$$

Assume that $H_{1(k)}^{(s, t)}+H_{2(k)}^{(s, t)} \neq 0$ for $1 \leqslant s<t \leqslant r-1$ and $k=1,2$. Let

$$
\Delta_{s t}=\sum_{k=1}^{2}\left(H_{1(k)}^{(s, t)}+H_{2(k)}^{(s, t)}\right)
$$

and

$$
H_{1(k)}^{*(s, t)}=\frac{H_{1(k)}^{(s, t)}}{\Delta_{s t}}, \quad H_{2(k)}^{*(s, t)}=\frac{H_{2(k)}^{(s, t)}}{\Delta_{s t}} \quad(k=1,2) .
$$

The MH model may be expressed as

$$
H_{1(k)}^{*(s, t)}=H_{2(k)}^{*(s, t)} \quad \text { for } \quad k=1,2 ; 1 \leqslant s<t \leqslant r-1 .
$$

Consider the measure $\Upsilon_{M H}$ defined by

$$
\Upsilon_{M H}=\frac{1}{\left(\begin{array}{c}
r-1 \\
2
\end{array}\right)} \sum_{1 \leqslant s<t \leqslant r-1} \sum_{s t}
$$

where

$$
\begin{aligned}
& \Upsilon_{s t}=\frac{4}{\pi} \sum_{k=1}^{2}\left(H_{1(k)}^{*(s, t)}+H_{2(k)}^{*(s, t)}\right)\left(\theta_{k}^{(s, t)}-\frac{\pi}{4}\right), \\
& \theta_{k}^{(s, t)}=\arccos \left(\frac{H_{1(k)}^{(s, t)}}{\sqrt{\left(H_{1(k)}^{(s, t)}\right)^{2}+\left(H_{2(k)}^{(s, t)}\right)^{2}}}\right) .
\end{aligned}
$$

The submeasure $\Upsilon_{s t}(1 \leqslant s<t \leqslant r-1)$ describes the degree of departure from marginal homogeneity for a collapsed table $T_{s t}$ with fixed $s$ and $t$ $(1 \leqslant s<t \leqslant r-1)$. The range of $\theta_{k}^{(s, t)}$ is $0 \leqslant \theta_{k}^{(s, t)} \leqslant \pi / 2$. Therefore, the ranges of $\Upsilon_{M H}$ and $\Upsilon_{s t}$ are between -1 and 1 .

The measure $\Upsilon_{M H}$ has the following characteristics: 
(i) $\Upsilon_{M H}=-1$ if and only if $H_{2(k)}^{(s, t)}=0$ for all $1 \leqslant s<t \leqslant r-1$ and $k=1,2$ (i.e., $p_{j i}=0$ for all $i<j(1 \leqslant i<j \leqslant r)$ ). Hereinafter, this is called L-marginal inhomogeneity;

(ii) $\Upsilon_{M H}=1$ if and only if $H_{1(k)}^{(s, t)}=0$ for all $1 \leqslant s<t \leqslant r-1$ and $k=1,2$ (i.e., $p_{i j}=0$ for all $i<j(1 \leqslant i<j \leqslant r)$ ). Hereinafter, this is called U-marginal inhomogeneity.

In addition, $\Upsilon_{M H}=0$ indicates that the weighted average of $\left(\theta_{k}^{(s, t)}-\right.$ $\pi / 4)$ is equal to 0 . Thus, $\Upsilon_{M H}=0$ indicates average marginal homogeneity. Note that if marginal homogeneity holds, then average marginal homogeneity holds. However, the converse is not the case.

$\Upsilon_{M H}$ indicates whether the departure from average marginal homogeneity is toward L-marginal inhomogeneity or U-marginal inhomogeneity. As $\Upsilon_{M H}$ approaches -1 , the departure from average marginal homogeneity becomes greater toward L-marginal inhomogeneity. On the other hand, as $\Upsilon_{M H}$ approaches 1, it becomes greater toward U-marginal inhomogeneity.

Furthermore, the submeasure $\Upsilon_{s t}$ for fixed $s$ and $t(1 \leqslant s<t \leqslant r-1)$ has the following characteristics.

(iii) $\Upsilon_{s t}=-1$ if and only if $H_{2(k)}^{(s, t)}=0$ for $k=1,2$. That is, L-marginal inhomogeneity holds in the collapsed table $T_{s t}$;

(iv) $\Upsilon_{s t}=1$ if and only if $H_{1(k)}^{(s, t)}=0$ for $k=1,2$. That is, U-marginal inhomogeneity holds in the collapsed table $T_{s t}$;

(v) $\Upsilon_{s t}=0$ if and only if average marginal homogeneity holds in the collapsed table $T_{s t}$.

$\Upsilon_{s t}$ indicates whether the departure from average marginal homogeneity is toward L-marginal inhomogeneity or U-marginal inhomogeneity in the collapsed table $T_{s t}$.

\section{Relationship between the measure and the extended marginal homogeneity model}

Consider the relationship between $\Upsilon_{M H}$ and the EMH model. The EMH model is defined as

$$
H_{1(k)}^{(s, t)}=\tau H_{2(k)}^{(s, t)}
$$


for $k=1,2$ and $1 \leqslant s<t \leqslant r-1$. When $\tau=1$, the EMH model is equivalent to the MH model. If the EMH model holds in an $r \times r$ table, both $\theta_{1}^{(s, t)}$ and $\theta_{2}^{(s, t)}$ in the measure $\Upsilon_{M H}$ can be expressed as

$$
\arccos \left(\frac{\tau}{\sqrt{\tau^{2}+1}}\right) \text {. }
$$

Namely, $\Upsilon_{M H}$ can be expressed as

$$
\Upsilon_{M H}=\frac{4}{\pi} \arccos \left(\frac{\tau}{\sqrt{\tau^{2}+1}}\right)-1 .
$$

Therefore, $\Upsilon_{M H}=0$ if and only if $\tau=1$. That is, marginal homogeneity holds. As $\tau$ approaches $\infty, \Upsilon_{M H}$ approaches -1, while the measure $\Upsilon_{M H}$ approaches 1 as $\tau$ approaches 0. Thus, when the EMH model holds in the table, $\Upsilon_{M H}$ represents the degree of departure from marginal homogeneity toward L-marginal inhomogeneity or U-marginal inhomogeneity.

Note that when the EMH model holds, the measure $\Upsilon_{M H}$ is equivalent to the measure $\Psi$ (Yamamoto et al., 2011) defined by

$$
\Psi=\frac{4}{\pi} \sum_{i=1}^{r-1}\left(G_{1(i)}^{*}+G_{2(i)}^{*}\right)\left(\theta_{i}-\frac{\pi}{4}\right)
$$

where

$$
\theta_{i}=\arccos \left(\frac{G_{1(i)}}{\sqrt{G_{1(i)}^{2}+G_{2(i)}^{2}}}\right)
$$

and

$$
\begin{aligned}
& G_{1(i)}=\sum_{s=1}^{i} \sum_{t=i+1}^{r} p_{s t}, \quad G_{2(i)}=\sum_{s=1}^{i} \sum_{t=i+1}^{r} p_{t s}, \\
& \Delta=\sum_{i=1}^{r-1}\left(G_{1(i)}+G_{2(i)}\right), \quad G_{1(i)}^{*}=\frac{G_{1(i)}}{\Delta}, \quad G_{2(i)}^{*}=\frac{G_{2(i)}}{\Delta}
\end{aligned}
$$

for $i=1, \ldots, r-1$.

Figure 1 shows the relationships between the parameter $\tau$ in the EMH model and the measures $\Upsilon_{M H}$ and $\Psi$ when the EMH model holds. 
In addition, if the EMH model holds in the collapsed table $T_{s t}, \Upsilon_{s t}$ can be expressed as

$$
\Upsilon_{s t}=\frac{4}{\pi} \arccos \left(\frac{\tau}{\sqrt{\tau^{2}+1}}\right)-1
$$

for fixed $s$ and $t(1 \leqslant s<t \leqslant r-1)$. Therefore, $\Upsilon_{s t}=0$ if and only if $\tau=1$. That is, marginal homogeneity holds in the collapsed table $T_{s t}$. If the EMH model holds in a collapsed table $T_{s t}, \Upsilon_{s t}$ represents the degree of departure from marginal homogeneity toward L-marginal inhomogeneity or U-marginal inhomogeneity in the collapsed table $T_{s t}$.

Figure 1. Relationships between $\tau$ in the EMH model and measures $\Upsilon_{M H}$ and $\Psi$ when the EMH model holds

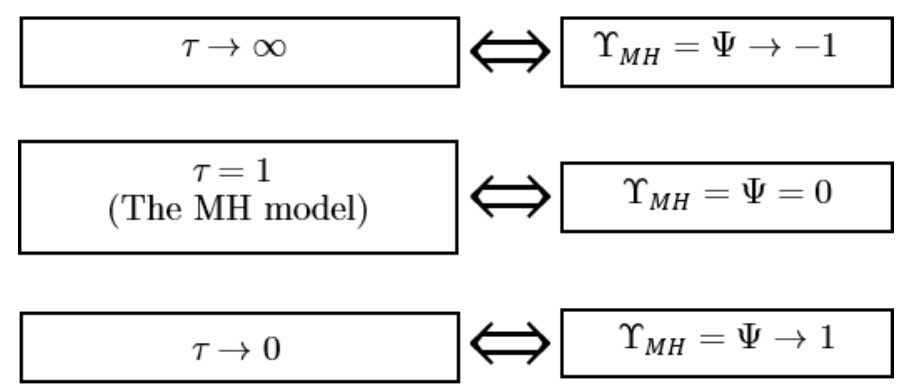

\section{Approximate confidence interval for the measure}

Let $n_{i j}$ denote the observed frequency in the $i$ th row and $j$ th column of an $r \times r$ table $(i=1, \ldots, r$ and $j=1, \ldots, r)$. Assuming that a multinomial distribution is applicable to the table, we consider the approximate variance for the estimated measure and a large-sample confidence interval for $\Upsilon_{M H}$ using the delta method. The sample version measure $\Upsilon_{M H}$ (i.e., $\hat{\Upsilon}_{M H}$ ) is given by $\Upsilon_{M H}$ when $\left\{p_{i j}\right\}$ is replaced by $\left\{\hat{p}_{i j}\right\}$, where $\hat{p}_{i j}=n_{i j} / n$ and $n=$ $\sum \sum n_{i j}$. Using the delta method, $\sqrt{n}\left(\hat{\Upsilon}_{M H}-\Upsilon_{M H}\right)$ has an asymptotically normal distribution (as $n \rightarrow \infty$ ) with mean 0 and variance $\sigma^{2}\left[\Upsilon_{M H}\right]$, where

$$
\sigma^{2}\left[\Upsilon_{M H}\right]=\sum_{i=1}^{r} \sum_{j=1}^{r} D_{i j}^{2} p_{i j}
$$


where

$$
\begin{aligned}
& D_{i j}=\frac{1}{\left(\begin{array}{c}
r-1 \\
2
\end{array}\right)} \sum_{s=1}^{r-2} \sum_{t=s+1}^{r-1}\left[\sum_{k=1}^{2}\left(A_{k}^{(s, t)} C_{i j(k)}^{(s, t)}+B_{k}^{(s, t)} C_{j i(k)}^{(s, t)}\right)\right], \\
& A_{k}^{(s, t)}=\frac{4}{\pi \Delta_{s t}}\left\{\theta_{k}^{(s, t)}-\frac{H_{1(k)}^{(s, t)}+H_{2(k)}^{(s, t)}}{\left(H_{1(k)}^{(s, t)}\right)^{2}+\left(H_{2(k)}^{(s, t)}\right)^{2}} H_{2(k)}^{(s, t)}\right\}-\frac{1+\Upsilon_{s t}}{\Delta_{s t}}, \\
& B_{k}^{(s, t)}=\frac{4}{\pi \Delta_{s t}}\left\{\theta_{k}^{(s, t)}+\frac{H_{1(k)}^{(s, t)}+H_{2(k)}^{(s, t)}}{\left(H_{1(k)}^{(s, t)}\right)^{2}+\left(H_{2(k)}^{(s, t)}\right)^{2}} H_{1(k)}^{(s, t)}\right\}-\frac{1+\Upsilon_{s t}}{\Delta_{s t}},
\end{aligned}
$$

and

$$
C_{i j(k)}^{(s, t)}= \begin{cases}I(1 \leqslant i \leqslant s, s+1 \leqslant j \leqslant r) & (k=1) \\ I(1 \leqslant i \leqslant t, t+1 \leqslant j \leqslant r) & (k=2)\end{cases}
$$

where $I(\cdot)$ is the indicator function. Note that $C_{i i(k)}^{(s, t)}=0$ for $k=1,2$.

For the submeasure $\Upsilon_{s t}$, using the delta method, $\sqrt{n}\left(\hat{\Upsilon}_{s t}-\Upsilon_{s t}\right)$ has an asymptotically normal distribution (as $n \rightarrow \infty$ ) with mean 0 and variance $\sigma^{2}\left[\Upsilon_{s t}\right]$, where

$$
\sigma^{2}\left[\Upsilon_{s t}\right]=\sum_{k=1}^{2}\left[\left(A_{k}^{(s, t)}\right)^{2} H_{1(k)}^{(s, t)}+\left(B_{k}^{(s, t)}\right)^{2} H_{2(k)}^{(s, t)}\right] .
$$

Let $\hat{\sigma}^{2}\left[\Upsilon_{M H}\right]$ denote $\sigma^{2}\left[\Upsilon_{M H}\right]$ when $\left\{p_{i j}\right\}$ is replaced by $\left\{\hat{p}_{i j}\right\}$. Namely, $\hat{\sigma}\left[\Upsilon_{M H}\right] / \sqrt{n}$ is the estimated standard error of $\hat{\Upsilon}_{M H}$. Moreover, $\hat{\Upsilon}_{M H} \pm$ $z_{p / 2} \hat{\sigma}\left[\Upsilon_{M H}\right] / \sqrt{n}$ is the approximate $100(1-p) \%$ confidence interval for $\Upsilon_{M H}$, where $z_{p / 2}$ is the percentage point from the standard normal distribution corresponding to a two-tail probability equal to $p$.

To evaluate the performance of the confidence interval for $\Upsilon_{M H}$, we demonstrate a simulation. Table 2(a), 2(b) and 2(c) show the artificial cell probability tables where the value of $\Upsilon_{M H}$ is equal to $0,-0.5,0.5$, respectively. For each experiment, the $95 \%$ confidence interval for $\Upsilon_{M H}$ was computed, and we checked whether it contained the true value of $\Upsilon_{M H}$. In each experiment, the samples are obtained from a population with the artificial cell probability tables in Table 2 . 
Table 2. Artificial cell probability tables

(a) $\Upsilon_{M H}=0$

\begin{tabular}{lllll}
\hline 0.05 & 0.08 & 0.02 & 0.03 & 0.02 \\
0.03 & 0.08 & 0.02 & 0.07 & 0.05 \\
0.04 & 0.03 & 0.07 & 0.05 & 0.01 \\
0.05 & 0.04 & 0.06 & 0.02 & 0.03 \\
0.03 & 0.02 & 0.03 & 0.03 & 0.04 \\
\hline
\end{tabular}

(b) $\Upsilon_{M H}=-0.5$

\begin{tabular}{lllll}
\hline 0.05 & 0.08 & 0.04 & 0.05 & 0.07 \\
0.04 & 0.03 & 0.03 & 0.08 & 0.05 \\
0.03 & 0.01 & 0.05 & 0.06 & 0.02 \\
0.01 & 0.06 & 0.03 & 0.01 & 0.08 \\
0.02 & 0.01 & 0.01 & 0.04 & 0.04 \\
\hline
\end{tabular}

(c) $\Upsilon_{M H}=0.5$

\begin{tabular}{lllll}
\hline 0.05 & 0.04 & 0.03 & 0.01 & 0.02 \\
0.08 & 0.03 & 0.01 & 0.06 & 0.01 \\
0.04 & 0.03 & 0.05 & 0.03 & 0.01 \\
0.05 & 0.08 & 0.06 & 0.01 & 0.04 \\
0.07 & 0.05 & 0.02 & 0.08 & 0.04 \\
\hline
\end{tabular}

Table 3 shows the coverage probability of 1,000,000 experiments with sample sizes of 100, 500, 1000 and 2000. Although it seems to be permissive when the sample size is 100, from Table 3 , we see that the coverage probabilities of the confidence interval for $\Upsilon_{M H}$ are close to $95 \%$ regardless of the value of the true $\Upsilon_{M H}$.

Table 3. Coverage probability of $95 \%$ confidence interval for $\Upsilon_{M H}$ of $1,000,000$

\begin{tabular}{cccc}
\multicolumn{4}{c}{ experiments } \\
\hline Sample size & $\Upsilon_{M H}=0$ & $\Upsilon_{M H}=-0.5$ & $\Upsilon_{M H}=0.5$ \\
\hline 100 & 0.931 & 0.933 & 0.933 \\
500 & 0.946 & 0.946 & 0.946 \\
1000 & 0.948 & 0.948 & 0.948 \\
2000 & 0.949 & 0.948 & 0.948 \\
\hline
\end{tabular}




\section{Examples}

Consider the data shown in Tables 1(a) and 1(b).

For Table 1(a), the values of the likelihood ratio chi-squared statistic $\left(G^{2}\right)$ for the MH and EMH models are 15.533 with 6 degrees of freedom (df) and 5.853 with $5 \mathrm{df}$, respectively. Thus, the MH model fits the data in Table 1(a) poorly; however, the EMH model fits the data in Table 1(a) well. For Table 1(b), the values of $G^{2}$ for the MH and EMH models are 7.929 with $6 \mathrm{df}$ and 5.882 with $5 \mathrm{df}$, respectively. Thus, the MH and EMH models fit the data in Table 1(b) well.

According to Table 4, the estimated value of $\Upsilon_{M H}$ applied in Table 1(a) is -0.257 , and the corresponding confidence interval does not include 0 . Therefore, the departure from average marginal homogeneity for the shift in $\mathrm{CrCl}$ in patients treated with telavancin is toward L-marginal inhomogeneity. This indicates that the $\mathrm{CrCl}$ value tends to be lower at the end of therapy than at the baseline in patients treated with telavancin.

Similarly, the estimated value of $\Upsilon_{M H}$ applied in Table 1(b) is 0.110, and the corresponding confidence interval includes 0 . Therefore, average marginal homogeneity may hold in the data for the shift in $\mathrm{CrCl}$ in patients treated with vancomycin.

In addition, the confidence intervals for $\Upsilon_{M H}$ for the data in Tables 1(a) and 1(b) do not overlap, demonstrating that the $\mathrm{CrCl}$ value from the baseline significantly differs in patients treated with telavancin and those treated with vancomycin.

We also analyze the data in Tables 1(a) and 1(b) using the submeasure $\Upsilon_{s t}(1 \leqslant s<t \leqslant 6)$ (Table 5). For Table $1(\mathrm{a})$, all the estimated submeasures and the corresponding confidence intervals are negative. Therefore, patients treated with telavancin tend to have a lower $\mathrm{CrCl}$ value at the end of therapy than at the baseline when the original seven categories are collapsed into three categories using any pattern.

For Table 1(b), the confidence intervals for the submeasures $\Upsilon_{12}, \Upsilon_{23}$ and $\Upsilon_{25}$ are positive and do not include 0 . From $\Upsilon_{12}$, patients treated with vancomycin tend to have a higher $\mathrm{CrCl}$ value at the end of therapy than at the baseline when the original 7 categories are collapsed into 3 categories as $(1) \leqslant 30,(2)>30-40$, and $(3)>40 \mathrm{~mL} /$ minute. A similar interpretation is obtained for $\Upsilon_{23}$ when the original 7 categories are collapsed into 3 categories as $(1) \leqslant 40,(2)>40-50$, and $(3)>50 \mathrm{~mL} /$ minute, and for $\Upsilon_{25}$ when the original 7 categories are collapsed into 3 categories as $(1) \leqslant 40$, 
(2) $>40-70$, and $(3)>70 \mathrm{~mL} /$ minute. Conversely, the confidence intervals for the other submeasures include 0 . Therefore, when we collapse the original 7 categories into 3 categories using the other patterns, average marginal homogeneity may hold in each collapsed table for Table 1(b).

Table 4. Estimates of the measure $\Upsilon_{M H}$, approximate standard errors for $\hat{\Upsilon}_{M H}$ and approximate $95 \%$ confidence intervals for $\Upsilon_{M H}$, applied to Tables 1(a) and

\begin{tabular}{cccc}
\multicolumn{4}{c}{$1(\mathrm{~b})}$. \\
\hline Applied data & Estimated measure & Standard error & Confidence interval \\
\hline Table 1(a) & -0.257 & 0.080 & $(-0.414,-0.101)$ \\
Table 1(b) & 0.110 & 0.082 & $(-0.052,0.271)$ \\
\hline
\end{tabular}

Table 5. Estimates of the submeasure $\Upsilon_{s t}$ and approximate $95 \%$ confidence intervals for $\Upsilon_{s t}$, applied to Tables 1(a) and 1(b).

\begin{tabular}{ccccc}
\hline & \multicolumn{2}{c}{ For Table 1(a) } & \multicolumn{2}{c}{ For Table 1(b) } \\
\cline { 2 - 5 } Submeasure & $\begin{array}{c}\text { Estimated } \\
\text { value }\end{array}$ & $\begin{array}{c}\text { Confidence } \\
\text { interval }\end{array}$ & $\begin{array}{c}\text { Estimated } \\
\text { value }\end{array}$ & $\begin{array}{c}\text { Confidence } \\
\text { interval }\end{array}$ \\
\hline$\Upsilon_{12}$ & -0.417 & $(-0.580,-0.255)$ & 0.230 & $(0.045,0.415)$ \\
$\Upsilon_{13}$ & -0.300 & $(-0.466,-0.135)$ & 0.155 & $(-0.027,0.337)$ \\
$\Upsilon_{14}$ & -0.331 & $(-0.498,-0.163)$ & 0.041 & $(-0.142,0.224)$ \\
$\Upsilon_{15}$ & -0.296 & $(-0.465,-0.128)$ & 0.101 & $(-0.079,0.280)$ \\
$\Upsilon_{16}$ & -0.321 & $(-0.499,-0.144)$ & 0.077 & $(-0.099,0.253)$ \\
$\Upsilon_{23}$ & -0.259 & $(-0.418,-0.101)$ & 0.212 & $(0.053,0.371)$ \\
$\Upsilon_{24}$ & -0.283 & $(-0.444,-0.123)$ & 0.120 & $(-0.041,0.280)$ \\
$\Upsilon_{25}$ & -0.255 & $(-0.416,-0.094)$ & 0.166 & $(0.008,0.324)$ \\
$\Upsilon_{26}$ & -0.271 & $(-0.440,-0.102)$ & 0.144 & $(-0.011,0.300)$ \\
$\Upsilon_{34}$ & -0.199 & $(-0.359,-0.040)$ & 0.072 & $(-0.086,0.230)$ \\
$\Upsilon_{35}$ & -0.175 & $(-0.334,-0.015)$ & 0.116 & $(-0.039,0.272)$ \\
$\Upsilon_{36}$ & -0.181 & $(-0.348,-0.014)$ & 0.098 & $(-0.056,0.251)$ \\
$\Upsilon_{45}$ & -0.193 & $(-0.355,-0.032)$ & 0.035 & $(-0.121,0.191)$ \\
$\Upsilon_{46}$ & -0.202 & $(-0.372,-0.032)$ & 0.019 & $(-0.134,0.173)$ \\
$\Upsilon_{56}$ & -0.174 & $(-0.344,-0.004)$ & 0.061 & $(-0.090,0.213)$ \\
\hline & & & &
\end{tabular}




\section{Concluding remarks}

The proposed measure $\Upsilon_{M H}$ represents the degree of departure from average marginal homogeneity and distinguishes between two kinds of complete marginal inhomogeneity (i.e., U-marginal inhomogeneity and L-marginal inhomogeneity). Since the measure $\hat{\Upsilon}_{M H}$ ranges from -1 to 1 regardless of the dimension and sample size, $\hat{\Upsilon}_{M H}$ is useful to compare the degrees of departure from average marginal homogeneity in several tables. In particular, when the EMH model holds in the table, $\hat{\Upsilon}_{M H}$ represents the degree of departure from marginal homogeneity toward either one of the complete marginal inhomogeneities.

As described above, considering collapsed $3 \times 3$ tables can simplify the interpretation. If various thresholds can be considered for clinical data, it is meaningful to equally assess square contingency tables in various collapsed patterns. Therefore, the measure $\Upsilon_{M H}$, which combines all submeasures $\Upsilon_{s t}$ with the same weights, may be useful to interpret such data.

\section{Acknowledgements}

The authors would like to thank the referees for their comments.

\section{REFERENCES}

Ando S. (2019): A bivariate index for visually measuring marginal inhomogeneity in square tables. International Journal of Statistics and Probability 8:58-65.

McCullagh P. (1977): A logistic model for paired comparisons with ordered categorical data. Biometrika 64: 449-453.

Nogid B., Lacy M. K., Jacobs M., Bruss J. and Dwyer J. (2018): Time course and extent of renal function changes in patients receiving treatment for staphylococcal pneumonias: an analysis comparing telavancin and vancomycin from the ATTAIN trials. Pharmacotherapy 38:990-998.

Stuart A. (1955): A test for homogeneity of the marginal distributions in a two-way classifications. Biometrika 42:412-416.

Tomizawa, S. (1984): Three kinds of decompositions for the conditional symmetry model in a square contingency table. Journal of the Japan Statistical Society $14: 35-42$.

Tomizawa S. and Makii T. (2001): Generalized measures of departure from marginal homogeneity for contingency tables with nominal categories. Journal of Statistical Research 35:1-24.

Tomizawa S., Miyamoto N. and Ashihara N. (2003): Measure of departure from marginal homogeneity for square contingency tables having ordered categories. Behaviormetrika 30:173-193. 
Yamamoto K., Ando S. and Tomizawa S. (2011): A measure of departure from average marginal homogeneity for square contingency tables with ordered categories. Revstat: Statistical Journal 9:115-126.

Yamamoto K., Iwama I. and Tomizawa S. (2020): Measure of departure from marginal homogeneity for the analysis of collapsed square contingency tables with ordered categories. Journal of Statistical Theory and Applications 19:212-222. 